\title{
Fractional View Analysis of Acoustic Wave Equations, Using Fractional-Order Differential Equations
}

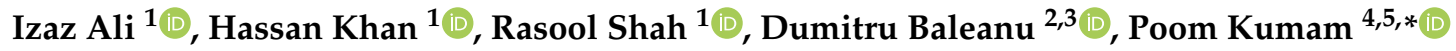 \\ and Muhammad Arif ${ }^{1}$ (D) \\ 1 Department of Mathematics, Abdul Wali Khan University, Mardan 23200, Pakistan; \\ izaz.ali@awkum.edu.pk (I.A.); hassanmath@awkum.edu.pk (H.K.); rasoolshah@awkum.edu.pk (R.S.); \\ marifmaths@awkum.edu.pk (M.A.) \\ 2 Department of Mathematics, Faculty of Arts and Sciences, Cankaya University, 06530 Ankara, Turkey; \\ dumitru@cankaya.edu.tr \\ 3 Institute of Space Sciences, 077125 Magurele, Romania \\ 4 Center of Excellence in Theoretical and Computational Science (TaCS-CoE) \& Department of Mathematics, \\ Faculty of Science, King Mongkut's University of Technology Thonburi (KMUTT), 126 Pracha Uthit Rd., \\ Bang Mod, Thung Khru, Bangkok 10140, Thailand \\ 5 Department of Medical Research, China Medical University Hospital, China Medical University, \\ Taichung 40402, Taiwan \\ * Correspondence: poom.kum@kmutt.ac.th
}

Received: 1 November 2019; Accepted: 15 December 2019; Published: 15 January 2020

\begin{abstract}
In the present research work, a newly developed technique which is known as variational homotopy perturbation transform method is implemented to solve fractional-order acoustic wave equations. The basic idea behind the present research work is to extend the variational homotopy perturbation method to variational homotopy perturbation transform method. The proposed scheme has confirmed, that it is an accurate and straightforward technique to solve fractional-order partial differential equations. The validity of the method is verified with the help of some illustrative examples. The obtained solutions have shown close contact with the exact solutions. Furthermore, the highest degree of accuracy has been achieved by the suggested method. In fact, the present method can be considered as one of the best analytical techniques compared to other analytical techniques to solve non-linear fractional partial differential equations.
\end{abstract}

Keywords: homotopy perturbation method; variational iteration method; Laplace transform method; acoustic wave equations

\section{Introduction}

Recently, fractional calculus and fractional differential equations (FDEs) have attracted the attention of scientists, mathematicians and engineers. A number of important implementations have been evaluated in various fields of sciences and engineering, such as material engineering, viscoelastic, electrochemistry, electromagnetic and dynamics physics which are described by fractional partial differential equations (FPDEs) [1]. Analytical approaches to solve FDEs are of great interest. There is no technique which provides an exact solution to the FDEs. Approximate approaches must be obtained by using techniques of series solution or linearization [2], followed by the application of proper numerical discretization [3-5] and system solvers [6-8]. Non-linear phenomena appear in a number of fields of engineering and sciences, such as solid state physics, chemical kinetics, non-linear spectroscopy, fluid physics, computational biology, quantum mechanics and thermodynamics etc. The concept of non-linearity is designed by various higher-order nonlinear partial differential equations (PDEs). For all of the physical systems, fundamental phenomena are covered by their nonlinear concepts $[9,10]$. 
In this paper, Laplace Variational Homotopy Perturbation Method (LVHPM) is implemented to solve the following linear and non-linear fractional-order regularized long wave equations.

$$
\frac{\partial^{\beta} v}{\partial \eta^{\beta}}+\frac{1}{2} \frac{\partial v^{2}}{\partial \xi}-\frac{\partial}{\partial \eta}\left(\frac{\partial^{2} v}{\partial \xi^{2}}\right)=0, \quad 0<\xi \leq 1, \quad 0<\beta \leq 1, \quad \eta>0
$$

with initial condition

$$
\begin{gathered}
v(\xi, 0)=\xi, \\
\frac{\partial^{\beta} v}{\partial \eta^{\beta}}+\frac{\partial v}{\partial \xi}+v \frac{\partial v}{\partial \eta}-\frac{\partial}{\partial \eta}\left(\frac{\partial^{2} v}{\partial \xi^{2}}\right)=0, \quad 0<\xi \leq 1, \quad 0<\beta \leq 1, \quad \eta>0,
\end{gathered}
$$

with initial condition

$$
\begin{gathered}
v(\xi, 0)=3 \alpha \operatorname{sech} h^{2}(\beta \xi), \quad \alpha>0, \quad \beta=\frac{1}{2} \sqrt{\frac{\alpha}{1+\alpha}} . \\
\frac{\partial^{\beta} v}{\partial \eta^{\beta}}+\frac{\partial v}{\partial \xi}-2 \frac{\partial}{\partial \eta}\left(\frac{\partial^{2} v}{\partial \xi^{2}}\right)=0, \quad 0<\xi \leq 1, \quad 0<\beta \leq 1, \quad \eta>0,
\end{gathered}
$$

with initial condition

$$
v(\xi, 0)=e^{-\xi}
$$

and

$$
\frac{\partial^{\beta} v(\xi \cdot \eta)}{\partial \eta^{\beta}}+\frac{\partial^{4} v(\xi \cdot \eta)}{\partial \xi^{4}}=0, \quad 0<\xi \leq 1, \quad 0<\beta \leq 1, \quad \eta>0,
$$

with initial condition

$$
v(\xi, 0)=\sin \xi .
$$

Equation (1) is known as the fractional-order non-linear regularized long wave equation (RLWE); Equation (2) is known as the fractional-order non-linear general regularized long wave equation (GRLWE) and Equations (3) and (4) are known as fractional-order linear regularized long wave equations (RLWEs) [11].

The Benjamin Bona Mahony equation (BBME) also identified the regularized long wave (RLW) equation. This equation is the updated version of Korteweg-de Vries equation $(\mathrm{KdV})$ for the modeling of tiny amplitude lengthy surface gravitational waves spreading unidirectionally in two dimensions. RLW equations have several implementations in certain areas of science, such as ion-acoustic waves in plasma, longitudinal dispersive waves in elastic rods, magneto-hydrodynamic waves in plasma, rotating tube flow and stress waves in compressed gas bubble mixes, etc. The RLW equations are described as useful models in applied physics and engineering for many significant physical structures. They also design many liquid flow nature issues where diffusion is significant, either in viscous or shock situations. It can be used to model any dissipation-related non-linear wave diffusion problem. Chemical reaction, heat conduction, mass diffusion, viscosity, thermal radiation or other sources may result from this dissipation, depending on problem modeling [12].

The RLW problem is a family of non-linear growth models that provides excellent designs for predicting natural phenomena. The algorithm was initially introduced to define the undular bore behavior [13]. It was also obtained from the research of acoustic plasma waves of water and ion. An analytical solution for the RLW equation was identified under restricted initial and boundary conditions in [14]. The fractional RLW equations also define numerous significant ocean science and engineering phenomena such as long-wave and small frequency shallow water waves. The non-linear waves modeled on the fractional equations of RLW are of significant interest for several scientists in ocean shallow waves of liquid. The mathematically modeled non-linear waves in the ocean were the fractional RLW equations. Indeed, huge surface waves identified as the tsunami are described as fractional RLW equations. The huge internal waves in the interior of the ocean, resulting from 
the difference in temperature, that may destruct marine ships could be defined as fractional RLW equations in the current, highly efficient method.

In recent decades, many researchers and scientists have used analytical methods to solve these types of problems such as homotopy perturbation Sumudu transform method (HPSTM) [11], Adomian decomposition method (ADM) [15,16], least-squares method [17], optimal homotopy perturbation method [18], variational iteration method (VIM) homotopy perturbation method (HPM) [19] and He's homotopy perturbation method [20]. It is observed that these methods have certain deficiencies like calculation of Adomian polynomials, determination of Lagrange multiplier, divergent results and a huge volume of calculations. As a result, a modified analytical technique which is known as VHPTM was introduced to solve differential equations of fractional-order. VHPTM is the combination of three well-known techniques namely, homotopy perturbation method, Laplace transform and variational iteration method. The present method uses the Lagrange multiplier that can limit the consecutive implementation of integral operator and unmanageable computational cost. It is still maintaining higher degree of accuracy. VHPTM [21-24] has an excellent scheme and absorbs all the beneficial characteristics of VIM and HPM.

Finally, He's polynomials have been used in the correction fractional formula to develop the homotopy perturbation method. It is observed that the proposed method is implemented without any use of transformation, discretization and it was found to be free from the generating round off error. Usually, the method of variable separable needs both initial and boundary points to operate, but the present method provides an analytical solution by using initial conditions only. There is a clear advantage of the suggested method that it works without any use of Adomian polynomials, as required by the Adomian decomposition method. Results of the analysis show that the suggested method produces the solution in a series of fast convergence that can result in a closed solution [25-30].

\section{Preliminaries Concepts}

Definition 1. Laplace transformation of $\rho(\eta), \eta>0$ represented as [13]

$$
Q(s)=\mathcal{L}[\rho(\eta)]=\int_{0}^{\infty} e^{-s \eta} \rho(\eta) d \eta .
$$

Theorem 1. The convolution of Laplace transform is

$$
\mathcal{L}\left[\rho_{1} \times \rho_{2}\right]=\mathcal{L}\left[\rho_{1}(\eta)\right] \times \mathcal{L}\left[\rho_{2}(\eta)\right],
$$

here $\rho_{1} \times \rho_{2}$, define the convolution between $\rho_{1}$ and $\rho_{2}$,

$$
\left(\rho_{1} \times \rho_{2}\right) \eta=\int_{0}^{\tau} \rho_{1}(\tau) \rho_{2}(\eta-\tau) d \eta .
$$

Laplace transform of fractional derivative

$$
\mathcal{L}\left(D_{\eta}^{\beta} \rho(\eta)\right)=s^{\beta} Q(s)-\sum_{k=0}^{n-1} s^{\beta-1-k} \rho^{(k)}(0), \quad n-1<\beta<n .
$$

where $Q(s)$ is the Laplace transformation of $\rho(\eta)$.

Definition 2. The Riemann-Liouville fractional integral operator of order $\beta \geq 0$ of a function $f \in C_{\mu}, \mu \geq-1$ is described in [21]

$$
I_{\xi}^{\beta} g(\xi)=\frac{1}{\Gamma(\beta)} \int_{0}^{\xi}(\xi-s)^{\beta-1} g(s) d s,
$$


where $\Gamma$ represent the gamma function as,

$$
\Gamma(\beta)=\int_{0}^{\infty} e^{-\xi} \xi^{\beta-1} d \xi \quad \beta \in \mathbb{C} .
$$

Definition 3. The fractional derivative of $g(\eta)$ in the Caputo sense is defined as

$$
D^{\beta} g(\eta)=\frac{\partial^{\beta} g(\eta)}{\partial \eta^{\beta}}=\left\{\begin{array}{l}
I^{m-\beta}\left[\frac{\partial^{m} g(\eta)}{\partial \eta^{m}}\right], \quad \text { if } m-1<\beta<m, \quad m \in \mathbb{N} \\
\frac{\partial^{m} g(\eta)}{\partial \eta^{m}}, \quad \beta=m .
\end{array}\right.
$$

Lemma 1. If $\tilde{m}-1<\beta \leq \tilde{m}$ with $\tilde{m} \in \mathbb{N}$ and $g \in \mathbb{C}_{\eta}$ with $\eta \geq-1$, then

$$
\begin{aligned}
& I^{\beta} I^{a} g(\xi)=I^{\beta+a} g(\xi), \quad a, \beta \geq 0 . \\
& I^{\beta} \xi^{\lambda}=\frac{\Gamma(\lambda+1)}{\Gamma(\gamma+\lambda+1)} \xi^{\beta+\lambda}, \quad \beta>0, \lambda>-1, \quad \xi>0 . \\
& I^{\beta} D^{\beta} g(\xi)=g(\xi)-\sum_{k=0}^{\tilde{m}-1} g^{(k)}\left(0^{+}\right) \frac{\xi^{k}}{k !}, \quad \text { for } \quad \xi>0, \tilde{m}-1<\beta \leq \tilde{m} .
\end{aligned}
$$

Definition 4. Function of Mittag-Leffler, $E_{\alpha, \beta}(\eta)$ for $\alpha, \beta>0$ is defined as

$$
E_{\alpha, \beta}(\eta)=\sum_{k=0}^{\infty} \frac{\eta^{k}}{\Gamma(k \alpha+\beta)}, \quad \alpha, \beta>0, \quad \eta \in \mathbb{C} .
$$

\section{The Procedure of VHPTM}

To demonstrate, the fundamental concept of the present method [21,22], we are considering

$$
D_{\eta}^{\beta} v(\xi, \eta)+\bar{R} v(\xi, \eta)+\bar{N} v(\xi, \eta)=f(\xi, \eta)
$$

with initial condition

$$
v(\xi, 0)=g(\xi),
$$

where $f(\xi, \eta)$ is an inhomogeneous term, $\bar{R}$ and $\bar{N}$ are particular linear and non-linear differential operators and $D_{\eta}^{\beta} v(\xi, \eta)$ is the Caputo fractional derivative of $v(\xi, \eta)$.

By taking Laplace transform of Equation (6) on both sides, we get

$$
£_{\eta}\{v(\xi, \eta)\}-\left.\sum_{k=0}^{m-1} s^{\beta-1-k} \frac{\partial^{k} v(\xi, \eta)}{\partial^{k} \eta}\right|_{t=0}=-£\{\bar{R} v(\xi, \eta)+\bar{N} v(\xi, \eta)-f(\xi, \eta)\},
$$

where $v(s)=£_{\eta}(v(\xi, \eta))=\int_{0}^{\infty} e^{-s \eta} v(\eta) d \eta$.

We can build a functional correction according to the variation iteration method

$$
£_{\eta}\left\{v_{j+1}(\xi, \eta)\right\}=£\left\{v_{j}(\xi, \eta)\right\}+\lambda(s)\left[s^{\beta} £\left\{v_{\eta}(\xi, \eta)-\left.\sum_{k=0}^{m-1} s^{\beta-1-k} \frac{\partial^{k} v(\xi, \eta)}{\partial^{k} \eta}\right|_{t=0}+£\{\bar{R} v(\xi, \eta)+\bar{N} v(\xi, \eta)-f(\xi, \eta)\}\right\}\right],
$$

where $\lambda(s)$ is the Lagrange multiplier. Here we put $\lambda(s)=\frac{-1}{s^{\beta}}$ [22].

Applying inverse Laplace of Equation (7)

$$
v_{j+1}(\xi, \eta)=v_{j}(\xi, \eta)-£^{-1}\left[\frac{1}{s^{\beta}} £\left\{s^{\beta} \frac{\partial v}{\partial \eta}+\bar{R} v_{j}(\xi, \eta)+\bar{N} v_{j}(\xi, \eta)-f(\xi, \eta)\right\}\right] .
$$


The basic idea in the procedure of homotopy perturbation method is that the solution can be written as a series in powers of $p$ :

$$
v(\xi, \eta)=\sum_{j=0}^{\infty} p^{j} v_{j}(\xi, \eta)=v_{0}+p v_{1}+p^{2} v_{2}+p^{3} v_{3}+\cdots,
$$

where the non-linear expression can be expressed as

$$
\bar{N} v(\xi, \eta)=\sum_{j=0}^{\infty} p^{j} \bar{H}_{j}(v)
$$

$\bar{H}_{j}$ is He's polynomials,

$$
\bar{H}_{j}\left(v_{0}+v_{1}+\cdots+u_{j}\right)=\frac{1}{j !} \frac{\partial^{j}}{\partial p^{j}}\left[\bar{N}\left(\sum_{i=0}^{\infty} p^{i} v_{i}\right)\right] .
$$

The technique of fractional VHPTM of Equation (8) with He's polynomials.

$$
\sum_{j=0}^{\infty} p^{j} v_{j}(\xi, \eta)=\sum_{j=0}^{\infty} p^{j} v_{j}(\xi, \eta)+£^{-1}\left[\lambda(s) £\left\{\sum_{j=0}^{\infty} p^{j} \frac{\partial^{\beta} v_{j}}{\partial \eta^{\beta}}(\xi, s)+\sum_{j=0}^{\infty} p^{j} \bar{R} v_{j}(\xi, \eta)+\sum_{j=0}^{\infty} p^{j} \bar{H}_{j}(v)-f(\xi, \eta)\right\}\right] .
$$

By comparing the coefficients of like power of $p$ on both sides of Equation (12), we get the VHPTM solution of the given problem.

Theorem 2. Let $\xi$ and $\mathcal{Y}$ be two Banach spaces and $T: \xi \rightarrow \mathcal{Y}$ be a contractive nonlinear operator, such that for all $v ; v^{*} \in ; \xi,\left\|T(v)-T\left(v^{*}\right)\right\| \leq K\left\|v-v^{*}\right\|, \quad 0<K<1$ [31]. Then, in view of Banach contraction theorem, $T$ has a unique fixed point $v$, such that $T v=v$ : Let us write the generated series (12), by the Laplace decomposition method as

$$
\xi_{m}=T\left(\xi_{m-1}\right), \quad \xi_{m-1}=\sum_{m=1}^{m-1} v_{j}, \quad m=0,1,2, \cdots
$$

and supposed that $\xi_{0}=v_{0} \in \mathcal{S}_{p}(v)$, where $\mathcal{S}_{p}(v)=\left\{v^{*} \in \xi:\left\|v-v^{*}\right\|<p\right\}$ then, we have

$$
\begin{aligned}
& \left(B_{1}\right) \xi_{m} \in \mathcal{S}_{p}(v) \\
& \left(B_{2}\right) \lim _{m \rightarrow \infty} \xi_{m}=v .
\end{aligned}
$$

Proof. $\left(B_{1}\right)$ In view of mathematical induction for $m=1$, we have

$$
\left\|\xi_{1}-v_{1}\right\|=\left\|T\left(\xi_{0}-T(v)\right)\right\| \leq K\left\|v_{0}-v\right\| .
$$

Let the result be true for $m-1$, then

$$
\left\|\xi_{m-1}-v\right\| \leq K^{m-1}\left\|v_{0}-v\right\| .
$$

We have

$$
\left\|\xi_{m}-v\right\|=\left\|T\left(\xi_{m-1}-T(v)\right)\right\| \leq K\left\|\xi_{m-1}-v\right\| \leq K^{m}\left\|v_{0}-v\right\| .
$$

Hence, using $\left(B_{1}\right)$, we have

$$
\left\|\xi_{m}-v\right\| \leq K^{m}\left\|v_{0}-v\right\| \leq K^{m} p<p,
$$

which implies that $\xi_{m} \in \mathcal{S}_{p}(v)$. 
$\left(B_{2}\right)$ : Since $\left\|\xi_{m}-v\right\| \leq K^{m}\left\|v_{0}-v\right\|$ and as a $\lim _{m \rightarrow \infty} K^{m}=0$.

Therefore; we have $\lim _{m \rightarrow \infty}\left\|\xi_{m}-v\right\|=0 \Rightarrow \lim _{m \rightarrow \infty} \xi_{m}=v$.

\section{Numerical Examples}

\subsection{Example}

We consider time fractional-order non-linear RLW equation

$$
\frac{\partial^{\beta} v}{\partial \eta^{\beta}}+\frac{1}{2} \frac{\partial v^{2}}{\partial \xi}-\frac{\partial \partial^{2} v}{\partial \eta \partial \xi^{2}}=0, \quad 0<\xi \leq 1, \quad 0<\beta \leq 1, \quad \eta>0,
$$

initial condition is

$$
v(\xi, 0)=\xi,
$$

By using Equation (12), the fractional PDE given in Equation (13) can be written as

$$
\sum_{j=0}^{\infty} p^{j} v_{j+1}(\xi, \eta)=\sum_{j=0}^{\infty} p^{j} v_{j}(\xi, \eta)+\epsilon^{-1}\left[\lambda(s) £\left\{s^{\beta} \frac{\partial v_{j}(\xi, \eta)}{\partial \eta}+\frac{1}{2} \frac{\partial v_{j}^{2}(\xi, \eta)}{\partial \xi}-\frac{\partial \partial^{2} v_{j}(\xi, \eta)}{\partial \eta \partial \xi^{2}}\right\}\right],
$$

where $\lambda(s)$ is the Lagrange multiplier

$$
\lambda(s)=\frac{-1}{s^{\beta}} .
$$

Applying VHPTM using He's polynomials,

$$
\begin{aligned}
& \sum_{j=0}^{\infty} p^{j} v_{j+1}(\xi, \eta)=\sum_{j=0}^{\infty} p^{j} v_{j}(\xi, \eta)-\sum_{j=0}^{\infty} p^{j} \epsilon^{-1}\left[\frac { 1 } { s ^ { \beta } } £ \left\{s^{\beta}\left(\frac{\partial v_{0}}{\partial \eta}+p \frac{\partial v_{1}}{\partial \eta}+p^{2} \frac{\partial v_{2}}{\partial \eta}+\cdots\right)\right.\right. \\
& \left.\left.+\frac{1}{2} \frac{\partial}{\partial \xi}\left\{v_{0}^{2}+p\left(2 v_{0} v_{1}\right)+p^{2}\left(2 v_{0} v_{2}+v_{1}^{2}\right)+\cdots\right\}-\left\{p^{0} \frac{\partial \partial^{2} v_{0}}{\partial \eta \partial \xi^{2}}+p^{1} \frac{\partial \partial^{2} v_{1}}{\partial \eta \partial \xi^{2}}+p^{2} \frac{\partial \partial^{2} v_{2}}{\partial \eta \partial \xi^{2}}+\cdots\right\}\right\}\right],
\end{aligned}
$$

Comparing the coefficients of $p$

$$
\begin{aligned}
& v_{0}(\xi, \eta)=\xi \\
& p^{1} v_{1}(\xi, \eta)=p^{1} v_{0}(\xi, \eta)-p^{1} £^{-1}\left[\frac{1}{s^{\beta}} £\left\{s^{\beta} \frac{\partial v_{0}(\xi, \eta)}{\partial \eta}+\frac{1}{2} \frac{\partial}{\partial \xi} v_{0}^{2}(\xi, \eta)-\frac{\partial \partial^{2} v_{0}(\xi, \eta)}{\partial \eta \partial \xi^{2}}\right\}\right], \\
& v_{1}(\xi, \eta)=\xi-\xi \frac{\eta^{\beta}}{\Gamma(\beta+1)}, \\
& p^{2} v_{2}(\xi, \eta)=p^{2} v_{1}(\xi, \eta)-p^{2} £^{-1}\left[\frac{1}{s^{\beta}} £\left\{s^{\beta} \frac{\partial v_{1}(\xi, \eta)}{\partial \eta}+\frac{1}{2} \frac{\partial}{\partial \xi}\left(2 v_{0} v_{1}\right)-\frac{\partial \partial^{2} v_{1}}{\partial \eta \partial \xi^{2}}\right\}\right] \\
& v_{2}(\xi, \eta)=\xi-\xi \frac{\eta^{\beta}}{\Gamma(\beta+1)}+2 \xi \frac{\eta^{2 \beta}}{\Gamma(2 \beta+1)}, \\
& p^{3} v_{3}(\xi, \eta)=p^{3} v_{2}(\xi, \eta)-p^{3} £^{-1}\left[\frac{1}{s^{\beta}} £\left\{s^{\beta} \frac{\partial v_{1}}{\partial \eta}+\frac{1}{2}\left(2 v_{0} v_{2}+v_{1}^{2}\right)-\frac{\partial \partial^{2} v_{2}}{\partial \eta \partial \xi^{2}}\right\}\right] \\
& v_{3}(\xi, \eta)=\xi-\xi \frac{\eta^{\beta}}{\Gamma(\beta+1)}+2 \xi \frac{\eta^{2 \beta}}{\Gamma(2 \beta+1)}-\xi \frac{\Gamma(2 \gamma+1) \eta^{3 \beta}}{(\Gamma(2 \gamma+1))^{2} \Gamma(3 \beta+1)}-4 \xi \frac{\eta^{3 \beta}}{\Gamma(3 \beta+1)}
\end{aligned}
$$


The analytical expression is therefore obtained in the following way

$$
v(\xi, \eta)=\xi-\xi \frac{\eta^{\beta}}{\Gamma(\beta+1)}+2 \xi \frac{\eta^{2 \beta}}{\Gamma(2 \beta+1)}-\xi \frac{\Gamma(2 \gamma+1) \eta^{3 \beta}}{(\Gamma(\gamma+1))^{2} \Gamma(3 \beta+1)}-4 \xi \frac{\eta^{3 \beta}}{\Gamma(3 \beta+1)}+\cdots
$$

If $\beta=1$ the series form is

$$
v(\xi, \eta)=\xi\left(1-\eta+\eta^{2}-\eta^{3}+\cdots\right) .
$$

The exact solution at $\beta=1$

$$
v(\xi, \eta)=\frac{\xi}{1+\eta} .
$$

Figure 1, shows the plot of exact and VHPTM solutions of example 4.1, at integer-order $\beta=1$. It is confirmed from the figure that both exact and VHPTM solutions are in good contact with each other. In Figure 2, the VHPTM solutions at different fractional-orders $\beta=1,0.8,0.6$ and 0.4 are calculated. Investigations show that the solutions at different fractional-orders are convergent to the solution of an integer problem as the fractional-order approaches to an integer-order.

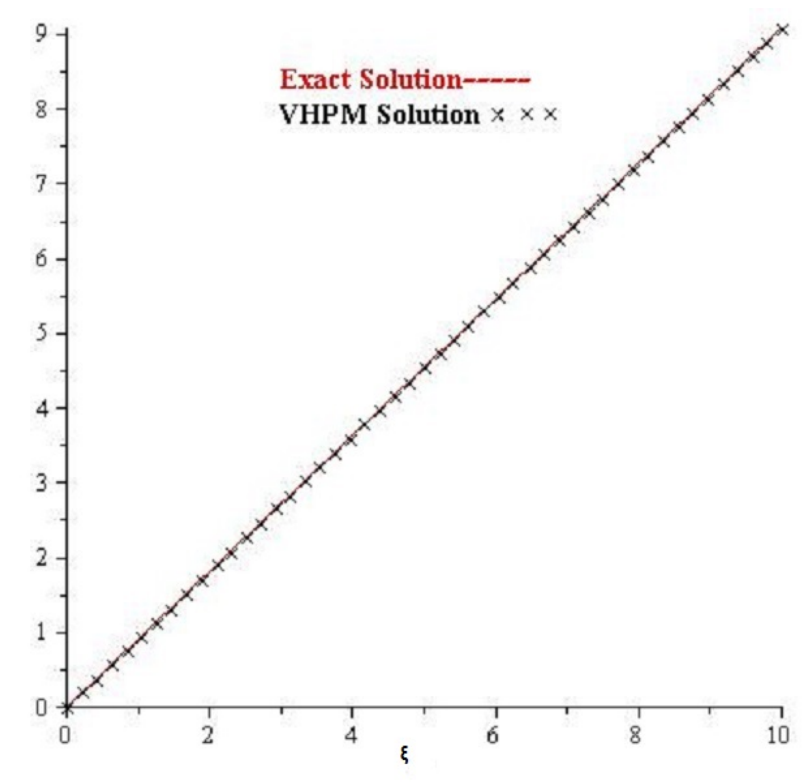

Figure 1. Variational homotopy perturbation transform method solution of example 4.1 for $\beta=1$.

In Table 1 , we compared the solutions of VHPTM and VIM at an integer-order $\beta=1$ for example 4.1. In addition, the solutions at fractional-orders $\beta=0.55$ and $\beta=0.75$ are listed in the table. It is observed that VHPTM solutions are almost identical with each other. The results given in the table support the applicability of the VHPTM. 


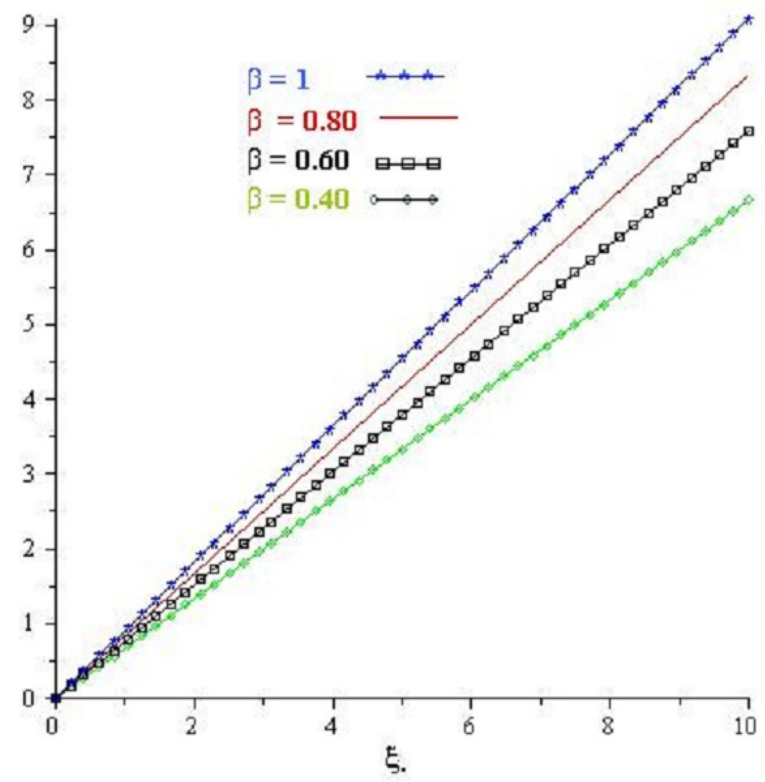

Figure 2. Variational homotopy perturbation transform method solution of example 4.1 at different fractional order $\beta=1,0.8,0.6,0.4$.

Table 1. Comparison of VHPTM and variational iteration method (VIM) [19] of example 1 at $\eta=0.1$.

\begin{tabular}{ccccccc}
\hline & VHPTM & VHPTM & VHPTM & Exact & Absolute Error & Absolute Error \\
\hline $\boldsymbol{\xi}$ & $\boldsymbol{\beta}=\mathbf{0 . 5 5}$ & $\boldsymbol{\beta}=\mathbf{0 . 7 5}$ & $\boldsymbol{\beta}=\mathbf{1}$ & & $\boldsymbol{V I M}(\boldsymbol{\beta}=\mathbf{1})$ & $\boldsymbol{V H P T M}(\boldsymbol{\beta}=\mathbf{1})$ \\
\hline 0.1 & 0.0966295721 & 0.0993928867 & 0.0999000999 & 0.0999001000 & $1 \times 10^{-10}$ & $1 \times 10^{-10}$ \\
0.2 & 0.1932591443 & 0.1987857735 & 0.1998001998 & 0.1998002000 & $2 \times 10^{-10}$ & $2 \times 10^{-10}$ \\
0.3 & 0.2898887165 & 0.2981786602 & 0.2997002997 & 0.2997003000 & $3 \times 10^{-10}$ & $3 \times 10^{-10}$ \\
0.4 & 0.3865182887 & 0.3975715470 & 0.3996003996 & 0.3996004000 & $4 \times 10^{-10}$ & $4 \times 10^{-10}$ \\
0.5 & 0.4831478608 & 0.4969644337 & 0.4995004995 & 0.4995005000 & $5 \times 10^{-10}$ & $5 \times 10^{-10}$ \\
0.6 & 0.5797774330 & 0.5963573204 & 0.5994005994 & 0.5994006000 & $6 \times 10^{-10}$ & $6 \times 10^{-10}$ \\
0.7 & 0.6764070052 & 0.6957502072 & 0.6993006993 & 0.6993007000 & $7 \times 10^{-10}$ & $7 \times 10^{-10}$ \\
0.8 & 0.7730365774 & 0.7951430939 & 0.7992007992 & 0.7992008000 & $8 \times 10^{-10}$ & $8 \times 10^{-10}$ \\
0.9 & 0.8696661495 & 0.8945359807 & 0.8991008991 & 0.8991009000 & $9 \times 10^{-10}$ & $9 \times 10^{-10}$ \\
1 & 0.9662957217 & 0.9939288674 & 0.9990009990 & 0.9990010000 & $10 \times 10^{-09}$ & $10 \times 10^{-09}$ \\
\hline
\end{tabular}

\subsection{Example}

We consider time fractional-order non-linear GRLW equation

$$
\frac{\partial^{\beta} v}{\partial \eta^{\beta}}+\frac{\partial v}{\partial \xi}+v \frac{\partial v}{\partial \xi}-\frac{\partial \partial^{2} v}{\partial \eta \partial \xi^{2}}=0, \quad 0<\xi \leq 1, \quad 0<\beta \leq 1, \quad \eta>0
$$

with initial condition

$$
v(\xi, 0)=3 \alpha \operatorname{sech}^{2}(\beta \xi), \quad \alpha>0, \quad \beta=\frac{1}{2} \sqrt{\frac{\alpha}{1+\alpha}} .
$$

By using Equation (12), the fractional PDE given in Equation (20) can be written as

$$
\sum_{j=0}^{\infty} p^{j} v_{j+1}(\xi, \eta)=\sum_{j=0}^{\infty} p^{j} v_{j}(\xi, \eta)+p^{j} £^{-1}\left[\lambda(s) £\left\{s^{\beta} \frac{\partial v_{j}(\xi, \eta)}{\partial \eta}+\frac{\partial v_{j}(\xi, \eta)}{\partial \xi}+v_{j}(\xi, \eta) \frac{\partial v_{j}(\xi, \eta)}{\partial \xi}-\frac{\partial \partial^{2} v_{j}(\xi, \eta)}{\partial \eta \partial \xi^{2}}\right\}\right]
$$

where $\lambda(s)$ is the Lagrange multiplier

$$
\lambda(s)=\frac{-1}{s^{\beta}} .
$$


Applying VHPTM using He's polynomials,

$$
\begin{aligned}
& \sum_{j=0}^{\infty} p^{j} v_{j}(\xi, \eta)=\sum_{j=0}^{\infty} p^{j} v_{j}(\xi, \eta)-p \epsilon^{-1}\left[\frac { 1 } { s ^ { \beta } } \epsilon \left\{s^{\beta}\left(\frac{\partial v_{0}}{\partial \eta}+p \frac{\partial v_{1}}{\partial \eta}+p^{2} \frac{\partial v_{1}}{\partial \eta}+\cdots\right)+\left\{\frac{\partial v_{0}}{\partial \xi}+p^{1} \frac{\partial v_{1}}{\partial \xi}+p^{2} \frac{\partial v_{2}}{\partial \xi}+\cdots\right\}\right.\right. \\
& \left.\left.+\left\{v_{0} \frac{\partial v_{0}}{\partial \xi}+p\left(v_{0} \frac{\partial v_{1}}{\partial \xi}+v_{1} \frac{\partial v_{0}}{\partial \xi}\right)+p^{2}\left(v_{0} \frac{\partial v_{2}}{\partial \xi^{\tau}}+v_{1} \frac{\partial v_{1}}{\partial \xi^{\tau}}+v_{2} \frac{\partial v_{0}}{\partial \xi}\right)+\cdots\right\}-\left\{\frac{\partial \partial^{2} v_{0}}{\partial \eta \partial \xi^{2}}+p^{1} \frac{\partial \partial^{2} v_{1}}{\partial \eta \partial \xi^{2}}+p^{2} \frac{\partial \partial^{2} v_{2}}{\partial \eta \partial \xi^{2}}+\cdots\right\}\right\}\right] .
\end{aligned}
$$

Comparing the coefficients of $p$

$$
\begin{aligned}
& v_{0}(\xi, \eta)=3 \alpha \sec h^{2}(\beta \xi), \\
& p^{1} v_{1}(\xi, \eta)=p^{1} v_{0}(\xi, \eta)-p^{1} £^{-1}\left[\frac{1}{s^{\beta}} £\left\{s^{\beta} \frac{\partial v_{0}}{\partial \eta}+\frac{\partial v_{0}}{\partial \xi}+v_{0} \frac{\partial v_{0}}{\partial \xi}-\frac{\partial \partial^{2} v_{0}}{\partial \eta \partial \xi^{2}}\right\}\right] \\
& v_{1}(\xi, \eta)=3 \alpha \sec h^{2}(\beta \xi)+3 \alpha \beta\{1+6 \alpha \beta+\cosh (2 \beta \xi)\} \sec h^{4}(\beta \xi) \tanh (\beta \xi) \frac{\eta^{\beta}}{\Gamma(\beta+1)}, \\
& p^{2} v_{2}(\xi, \eta)=p^{2} v_{1}(\xi, \eta)-p^{2} £^{-1}\left[\frac{1}{s^{\beta}} £\left\{s^{\beta} \frac{\partial v_{1}}{\partial \eta}+\frac{\partial v_{1}}{\partial \xi}+v_{0} \frac{\partial v_{1}}{\partial \xi}+v_{1} \frac{\partial v_{0}}{\partial \xi}-\frac{\partial \partial^{2} v_{1}}{\partial \eta \partial \xi^{2}}\right\}\right] \\
& v_{2}(\xi, \eta)=3 \alpha \sec h^{2}(\beta \xi)+3 \alpha \beta\{1+6 \alpha \beta+\cosh (2 \beta \xi)\} \sec h^{4}(\beta \xi) \tanh (\beta \xi) \frac{\eta^{\beta}}{\Gamma(\beta+1)} \\
& -\frac{3}{32} \alpha \beta^{2}\left\{-8-96 \alpha-576 \alpha^{2}+3\left(-3-16 \alpha+144 \alpha^{2}\right) \cosh (2 \beta \xi)+48 \alpha \cosh (4 \beta \xi)+\cosh (6 \beta \xi)\right\} \\
& \sec h^{8}(\beta \xi) \frac{\eta^{2 \beta}}{\Gamma(2 \beta+1)}
\end{aligned}
$$

The analytical expression is therefore obtained in the following way

$$
\begin{aligned}
& v(\xi, \eta)=3 \alpha \sec h^{2}(\beta \xi)+3 \alpha \beta\{1+6 \alpha \beta+\cosh (2 \beta \xi)\} \sec h^{4}(\beta \xi) \tanh (\beta \xi) \frac{\eta^{\beta}}{\Gamma(\beta+1)} \\
& -\frac{3}{32} \alpha \beta^{2}\left\{-8-96 \alpha-576 \alpha^{2}+3\left(-3-16 \alpha+144 \alpha^{2}\right) \cosh (2 \beta \xi)+48 \alpha \cosh (4 \beta \xi)+\cosh (6 \beta \xi)\right\} \\
& \sec h^{8}(\beta \xi) \frac{\eta^{2 \beta}}{\Gamma(2 \beta+1)}+\frac{1}{32} \alpha \beta^{3}\left\{-85-1416 \alpha-8496 \beta^{2}-2937 \beta^{3}+4\left(-31-432 \alpha-1584 \alpha^{2}+3456 \alpha^{3}\right)\right. \\
& \cosh (2 \beta \xi)-4\left(11+54 \alpha-540 \alpha^{2}\right) \cosh (4 \beta \xi)-4 \cosh (6 \beta \xi)+96 \alpha \cosh (6 \beta \xi)+\cosh (8 \beta \xi)+\sec h^{8}(\beta \xi) \\
& \tanh (\beta \xi)\} \frac{\eta^{3 \beta}}{\Gamma(3 \beta+1)}+\cdots
\end{aligned}
$$

The exact solution at $\beta=1$

$$
v(\xi, \eta)=3 \alpha \sec h^{2}(\beta(\xi-(1+\alpha) \eta)) .
$$

In Figure 3, we compared the analytical solution of VHPTM with the exact solution of example 4.2. The comparison has shown the close contact between VHPTM solution and exact solution of the problems. Figure 4, represents VHPTM solution at different fractional-orders $\beta=1,0.8,0.6$ and 0.4 The convergence analysis of fractional-order problems are convergent towards the integer-order problem of example 4.2, as observed. 


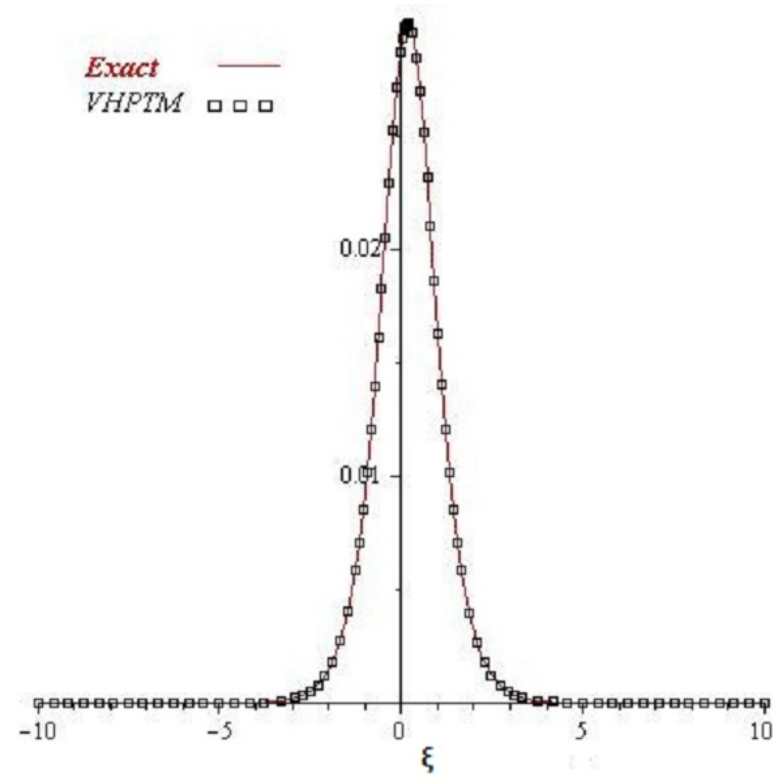

Figure 3. Variational homotopy perturbation transform method solution of example 4.2 for $\beta=1$.

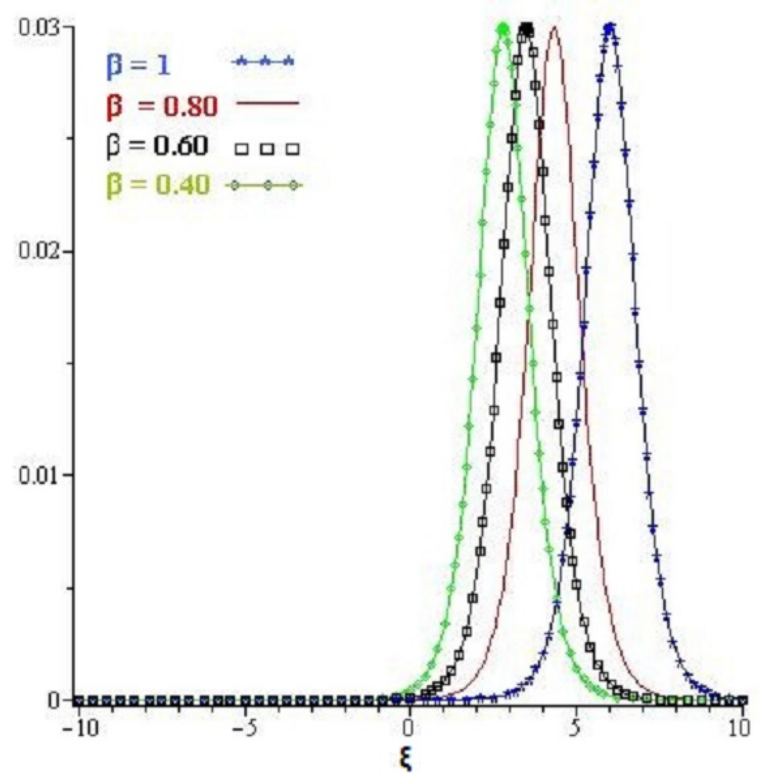

Figure 4. Variational homotopy perturbation transform method solution of example 4.2 at different fractional order $\beta=1,0.8,0.6,0.4$.

\subsection{Example}

We consider time fractional-order linear RLW equation

$$
\frac{\partial^{\beta} v}{\partial \eta^{\beta}}+\frac{\partial v}{\partial \xi}-2 \frac{\partial \partial^{2} v}{\partial \eta \partial \xi^{2}}=0, \quad 0<\xi \leq 1, \quad 0<\beta \leq 1, \quad \eta>0
$$

initial condition is

$$
v(\xi, 0)=e^{-\xi},
$$


By using Equation (12), the fractional PDE given in Equation (26) can be written as

$$
\sum_{j=0}^{\infty} p^{j} v_{j+1}(\xi, \eta)=\sum_{j=0}^{\infty} p^{j} v_{j}(\xi, \eta)+p^{j} \mathcal{E}^{-1}\left[\lambda(s) £\left\{s^{\beta} \frac{\partial^{\beta} v_{j}}{\partial \eta^{\beta}}(\xi, \eta)+\frac{\partial v_{j}}{\partial \xi}-2 \frac{\partial \partial^{2} v_{j}}{\partial \eta \partial \xi^{2}}\right\}\right],
$$

where $\lambda(s)$ is the Lagrange multiplier

$$
\lambda(s)=\frac{-1}{s^{\beta}} .
$$

Applying VHPTM using He's polynomials,

$$
\begin{aligned}
& \sum_{j=0}^{\infty} p^{j} v_{j}(\xi, \eta)=\sum_{j=0}^{\infty} p^{j} v_{j}(\xi, \eta)-p \AA^{-1}\left[\frac { 1 } { s ^ { \beta } } \notin \left\{s^{\beta}\left(\frac{\partial v_{0}}{\partial \eta}+p \frac{\partial v_{1}}{\partial \eta}+p^{2} \frac{\partial v_{2}}{\partial \eta}+\cdots\right)\right.\right. \\
& \left.\left.+\frac{\partial}{\partial \xi}\left\{v_{0}+p v_{1}+p^{2} v_{2}+\cdots\right\}-2\left\{\frac{\partial \partial^{2} v_{0}}{\partial \eta \partial \xi^{2}}+p^{1} \frac{\partial \partial^{2} v_{1}}{\partial \eta \partial \xi^{2}}+p^{2} \frac{\partial \partial^{2} v_{2}}{\partial \eta \partial \xi^{2}}+\cdots\right\}\right\}\right] .
\end{aligned}
$$

Comparing the coefficients of $p$

$$
\begin{aligned}
& v_{0}(\xi, \eta)=e^{-\xi} \\
& p^{1} v_{1}(\xi, \eta)=p^{1} v_{0}(\xi, \eta)-p^{1} £^{-1}\left[\frac{1}{s^{\beta}} £\left\{s^{\beta} \frac{\partial v_{0}}{\partial \eta}(\xi, \eta)+\frac{\partial v_{0}}{\partial \xi}-2 \frac{\partial \partial^{2} v_{0}}{\partial \eta \partial \xi^{2}}\right\}\right], \\
& v_{1}(\xi, \eta)=e^{-\xi}+e^{-\xi} \frac{\eta^{\beta}}{\Gamma(\beta+1)}, \\
& p^{2} v_{2}(\xi, \eta)=p^{2} v_{1}(\xi, \eta)-p^{2} £^{-1}\left[\frac{1}{s^{\beta}} £\left\{s^{\beta} \frac{\partial v_{1}}{\partial \eta}(\xi, \eta)+\frac{\partial v_{1}}{\partial \xi}-2 \frac{\partial \partial^{2} v_{1}}{\partial \eta \partial \xi^{2}}\right\}\right], \\
& v_{2}(\xi, \eta)=e^{-\xi}+e^{-\xi} \frac{\eta^{\beta}}{\Gamma(\beta+1)}+e^{-\xi} \frac{\eta^{2 \beta}}{\Gamma(2 \beta+1)}, \\
& p^{3} v_{3}(\xi, \eta)=p^{3} v_{2}(\xi, \eta)-p^{3} £^{-1}\left[\frac{-1}{s^{\beta}} £\left\{s^{\beta} \frac{\partial v_{2}}{\partial \eta}(\xi, \eta)+\frac{\partial v_{2}}{\partial \xi}-2 \frac{\partial \partial^{2} v_{2}}{\partial \eta \partial \xi^{2}}\right\}\right], \\
& v_{3}(\xi, \eta)=e^{-\xi}+e^{-\xi} \frac{\eta^{\beta}}{\Gamma(\beta+1)}+e^{-\xi} \frac{\eta^{2 \beta}}{\Gamma(2 \beta+1)}+e^{-\xi} \frac{\eta^{3 \beta}}{\Gamma(3 \beta+1)} .
\end{aligned}
$$

The analytical expression is therefore obtained in the following way

$$
v(\xi, \eta)=e^{-\xi}+e^{-\xi} \frac{\eta^{\beta}}{\Gamma(\beta+1)}+e^{-\xi} \frac{\eta^{2 \beta}}{\Gamma(2 \beta+1)}+e^{-\tau} \frac{\eta^{3 \beta}}{\Gamma(3 \beta+1)}+\cdots
$$

If $\beta=1$ the series form is

$$
v(\xi, \eta)=e^{-\xi}\left(1+\eta+\frac{\eta^{2}}{2 !}+\frac{\eta^{3}}{3 !}+\cdots\right)
$$

The exact solution at $\beta=1$

$$
v(\xi, \eta)=e^{\eta-\xi} .
$$

In Table 2, the analytical solutions of VHPTM and HPSTM are compared in terms of absolute error. The accuracy has been measured for both the methods. By comparison it has shown that the proposed method VHPTM has a higher degree of accuracy than HPSTM. 
In Figure 5, the graphs of exact and approximate solutions of example 4.3 are plotted. The graphical representation has confirmed that exact and VHPTM solutions are coincident. The exact and approximate solutions are closed to each other and verify the validity of the proposed method. The solution of example 4.3 at different fractional-orders $\beta=1,0.8,0.6$ and 0.4 are shown graphically in Figure 6. The obtained solutions support the convergence phenomena of the solution of fractional-order problems to the solution of integer-order problem for the example 4.3.

Table 2. Comparison of VHPTM and HPSTM [11] of example 4.3 at $\eta=0.0000001$.

\begin{tabular}{ccccc}
\hline & VHPTM & Exact & Absolute Error & Absolute Error \\
\hline $\boldsymbol{\zeta}$ & $\boldsymbol{\beta}=\mathbf{1}$ & & $\boldsymbol{H P S T M}(\boldsymbol{\beta}=\mathbf{1})$ & $\boldsymbol{V H \boldsymbol { H T M }}(\boldsymbol{\beta}=\mathbf{1})$ \\
\hline 0.1 & 0.9048374271 & 0.9048374180 & $8.396197700 \times 10^{-09}$ & $9.1000000 \times 10^{-10}$ \\
0.2 & 0.8187307613 & 0.8187307531 & $2.343247660 \times 10^{-09}$ & $8.2000000 \times 10^{-09}$ \\
0.3 & 0.7408182281 & 0.7408182207 & $1.098518064 \times 10^{-08}$ & $7.4000000 \times 10^{-09}$ \\
0.4 & 0.6703200527 & 0.6703200460 & $2.435301980 \times 10^{-08}$ & $6.7000000 \times 10^{-09}$ \\
0.5 & 0.6065306658 & 0.6065306597 & $3.925301546 \times 10^{-08}$ & $6.1000000 \times 10^{-09}$ \\
0.6 & 0.5488116416 & 0.5488116361 & $5.222691016 \times 10^{-08}$ & $5.5000000 \times 10^{-09}$ \\
0.7 & 0.4965853088 & 0.4965853038 & $6.061930396 \times 10^{-08}$ & $5.0000000 \times 10^{-09}$ \\
0.8 & 0.4493289686 & 0.4493289641 & $6.351737693 \times 10^{-08}$ & $4.5000000 \times 10^{-09}$ \\
0.9 & 0.4065696638 & 0.4065696597 & $6.159048138 \times 10^{-08}$ & $4.1000000 \times 10^{-09}$ \\
1 & 0.3678794449 & 0.3678794412 & $5.612475460 \times 10^{-08}$ & $3.7000000 \times 10^{-09}$ \\
\hline
\end{tabular}

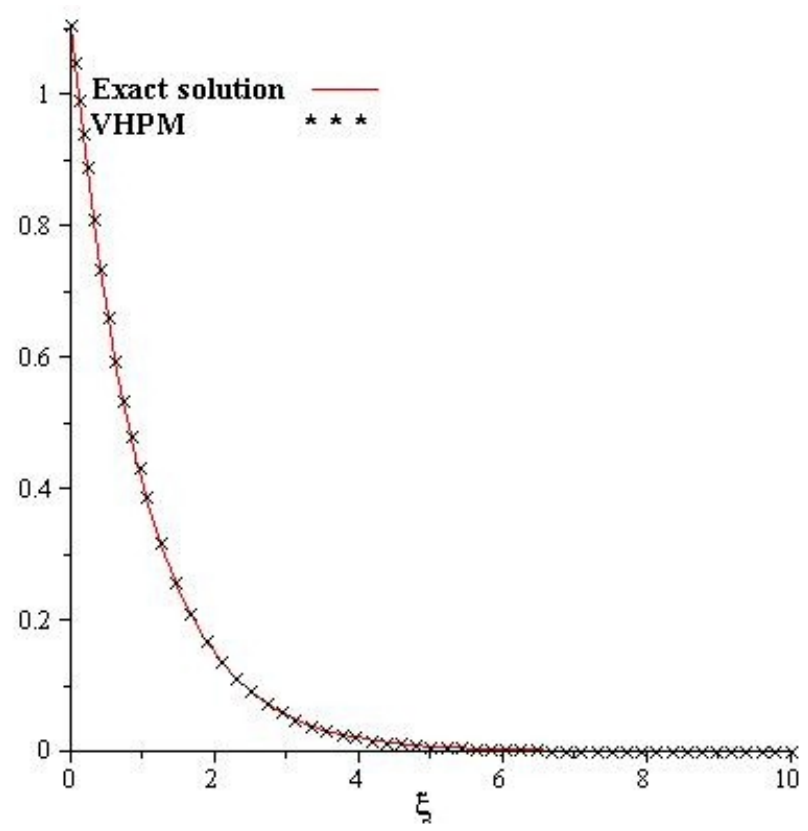

Figure 5. Variational homotopy perturbation transform method solution of example 4.3 for $\beta=1$. 


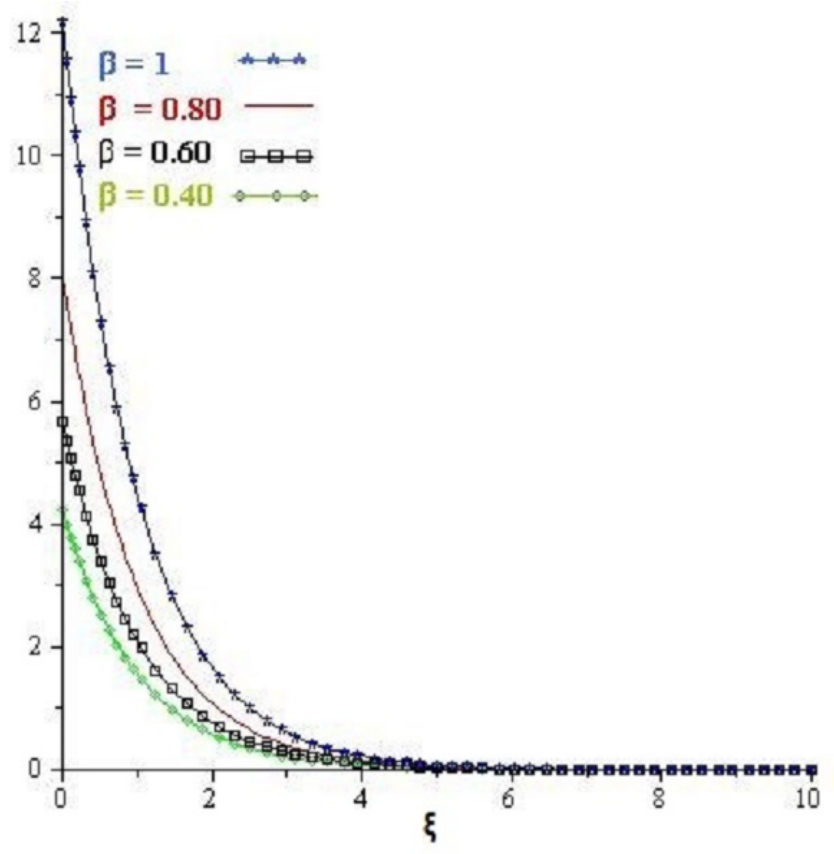

Figure 6. Variational homotopy perturbation transform method solution of example 4.3 at different fractional order $\beta=1,0.8,0.6,0.4$.

\subsection{Example}

We consider time fractional-order linear RLW equation

$$
\frac{\partial^{\beta} v(\xi \cdot \eta)}{\partial \eta^{\beta}}+\frac{\partial^{4} v(\xi \cdot \eta)}{\partial \xi^{4}}=0, \quad 0<\xi \leq 1, \quad 0<\beta \leq 1, \quad \eta>0,
$$

with initial condition

$$
v(\xi, 0)=\sin \xi,
$$

By using Equation (12), the fractional PDE given in Equation (33) can be written as

$$
\sum_{j=0}^{\infty} p^{j} v_{j+1}(\xi, \eta)=\sum_{j=0}^{\infty} p^{j} v_{j}(\xi, \eta)+\sum_{j=0}^{\infty} p^{j} £^{-1}\left[\lambda(s) £\left\{s^{\beta} \frac{\partial^{\beta} v_{j}(\xi \cdot \eta)}{\partial \eta^{\beta}}(\xi, \eta)+\frac{\partial^{4} v_{j}(\xi \cdot \eta)}{\partial \xi^{4}}\right\}\right],
$$

where $\lambda(s)$ is the Lagrange multiplier

$$
\lambda(s)=\frac{-1}{s^{\beta}},
$$

Applying VHPTM using He's polynomials,

$$
\begin{aligned}
& \sum_{j=0}^{\infty} p^{j} v_{j}(\xi, \eta)=\sum_{j=0}^{\infty} p^{j} v_{j}(\xi, \eta)-p £^{-1}\left[\frac { 1 } { s ^ { \beta } } £ \left\{s^{\beta}\left(\frac{\partial v_{0}}{\partial \eta}+p \frac{\partial v_{1}}{\partial \eta}+p^{2} \frac{\partial v_{2}}{\partial \eta}+\cdots\right)\right.\right. \\
& \left.\left.+\frac{\partial^{4}}{\partial \xi^{4}}\left\{v_{0}+p v_{1}+p^{2} v_{2}+\cdots\right\}\right\}\right]
\end{aligned}
$$

Comparing the coefficients of $p$ 


$$
\begin{aligned}
& v_{0}(\xi, \eta)=\sin \xi \\
& p^{1} v_{1}(\xi, \eta)=p^{1} v_{0}(\xi, \eta)-p^{1} £^{-1}\left[\frac{1}{s^{\beta}} £\left\{s^{\beta} \frac{\partial v_{0}(\xi \cdot \eta)}{\partial \eta}(\xi, \eta)+\frac{\partial^{4} v_{0}(\xi \cdot \eta)}{\partial \xi^{4}}\right\}\right], \\
& v_{1}(\xi, \eta)=\sin \xi-\sin \xi \frac{\eta^{\beta}}{\Gamma(\beta+1)}, \\
& p^{2} v_{2}(\xi, \eta)=p^{2} v_{1}(\xi, \eta)-p^{2} £^{-1}\left[\frac{1}{s^{\beta}} £\left\{s^{\beta} \frac{\partial v_{1}(\xi \cdot \eta)}{\partial \eta}(\xi, \eta)+\frac{\partial^{4} v_{1}(\xi \cdot \eta)}{\partial \xi^{4}}\right\}\right], \\
& v_{2}(\xi, \eta)=\sin \xi-\sin \xi \frac{\eta^{\beta}}{\Gamma(\beta+1)}+\sin \xi \frac{\eta^{2 \beta}}{\Gamma(2 \beta+1)}, \\
& p^{3} v_{3}(\xi, \eta)=p^{3} v_{2}(\xi, \eta)-p^{3} £^{-1}\left[\frac{1}{s^{\beta}} £\left\{s^{\beta} \frac{\partial v_{2}(\xi \cdot \eta)}{\partial \eta}(\xi, \eta)+\frac{\partial^{4} v_{2}(\xi \cdot \eta)}{\partial \xi^{4}}\right\}\right], \\
& v_{3}(\xi, \eta)=\sin \xi-\sin \xi \frac{\eta^{\beta}}{\Gamma(\beta+1)}+\sin \xi \frac{\eta^{2 \beta}}{\Gamma(2 \beta+1)}-\sin \xi \frac{\eta^{3 \beta}}{\Gamma(3 \beta+1)} .
\end{aligned}
$$

The analytical expression is therefore obtained in the following way

$$
v(\xi, \eta)=\sin \xi-\sin \xi \frac{\eta^{\beta}}{\Gamma(\beta+1)}+\sin \xi \frac{\eta^{2 \beta}}{\Gamma(2 \beta+1)}-\sin \xi \frac{\eta^{3 \beta}}{\Gamma(3 \beta+1)}+\cdots
$$

If $\beta=1$ the series form is

$$
v(\xi, \eta)=\sin \xi\left(1-\eta+\frac{\eta^{2}}{2 !}-\frac{\eta^{3}}{3 !}+\cdots\right) .
$$

The exact solution at $\beta=1$

$$
v(\xi, \eta)=\sin \xi e^{-\eta} .
$$

In Figure 7, the exact and VHPTM solution for example 4.4 are plotted. It can be seen from the figure that exact and VHPTM solutions are in closed contact with each other. In Figure 8, the VHPTM solutions for the example 4.4 at different fractional-orders are calculated. The convergence of fractional-order solutions towards integer-order solution has proved the applicability of the proposed method.

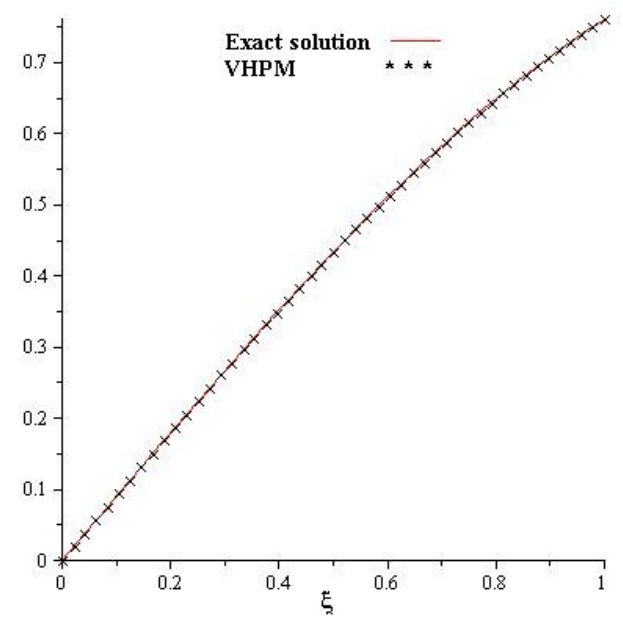

Figure 7. Variational homotopy perturbation transform method solution of example 4.4 for $\beta=1$. 


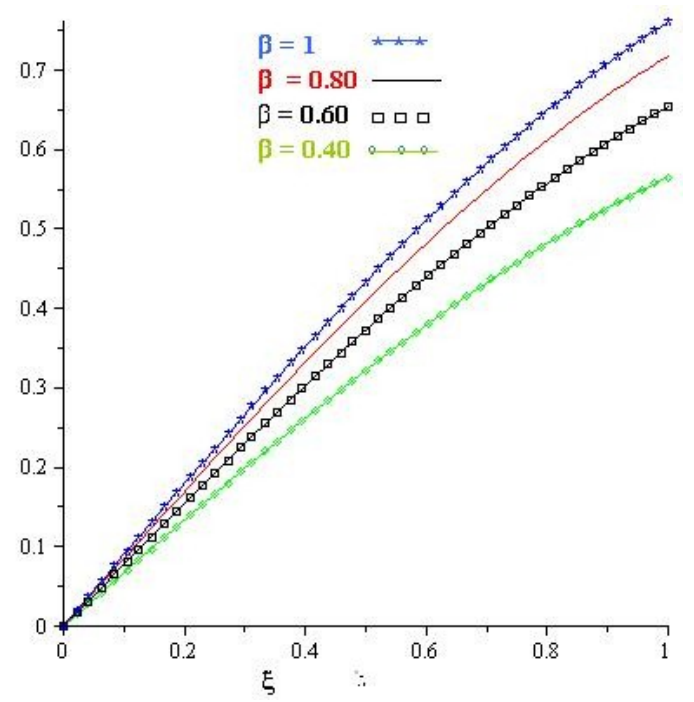

Figure 8. Variational homotopy perturbation transform method solution of example 4.4 at different fractional order $\beta=1,0.8,0.6,0.4$.

\section{Results and Discussion}

Several numerical examples are considered checking the applicability and reliability of the proposed method. The graphical representations of the solutions of examples 1 to 4 , have provided the information about the accuracy and reliability of the proposed method. All the results of examples $1-4$, confirmed strong agreement of VHPTM solutions with the exact solutions of the problems. The graphs represented the solutions for each problems at different fractional-order $\beta=1,0.8,0.6$ and 0.4. Investigations show that solutions of fractional-order problem are convergent to the solution of integer-order problems. Moreover, the simple and straightforward implementation of the suggested method is also observed throughout the simulation. From the above properties of the present method, we expect that it can be modified for other fractional-order differential equations which arise in science and engineering.

\section{Conclusions}

In this article, the fractional view of acoustic wave equation is discussed by using a modified analytical technique. The solution graphs are plotted to provide clear pictures and analysis of the obtained results. The graphical representation has suggested the greatest rate of convergence as compared to other analytical methods. The fractional-order analysis of the acoustic wave equation is important to investigate the behaviour of the dynamics as compared to the classical one. Therefore, in the present application scenario, the proposed method has played a significant role to describe sophisticated solutions of fractional-order partial differential equations arising in different areas of sciences and engineering. Moreover, the present method uses the variational parameters which reduces the calculations' complexity. Also, the He's polynomials have been used to obtain the solutions in an accurate way as compared to Adomian polynomials. The rate of convergence of the suggested method is found to be higher than other existing methods. Hence, it is concluded that the present method can be extended to solve other fractional non-linear partial differential equations.

Author Contributions: Conceptualization, I.A. and H.K.; Methodology, R.S.; Software, H.K.; Validation, R.S. and I.A.; Formal Analysis, R.S.; Investigation, R.S. and D.B.; Resources, H.K. and P.K.; Writing-Original Draft Preparation, R.S.; Writing-Review and Editing, H.K., M.A. and P.K.; Visualization, M.A.; Supervision, D.B., P.K.; Project Administration, P.K.; Funding Acquisition, P.K. All authors have read and agreed to the published version of the manuscript.

Funding: Center of Excellence in Theoretical and Computational Science (TaCS-CoE) Faculty of Science, King Mongkuts University of Technology Thonburi (KMUTT). 
Conflicts of Interest: The authors declare no conflict of interest.

\section{References}

1. Debnath, L. Recent applications of fractional calculus to science and engineering. Int. J. Math. Math. Sci. 2003, 54, 3413-3442. [CrossRef]

2. Miller, K.S.; Ross, B. An Introduction to the Fractional Calculus and Fractional Differential Equations; Wiley: New York, NY, USA, 1993.

3. Meerschaert, M.M.; Tadjeran, C. Finite difference approximations for two-sided space-fractional partial differential equations. Appl. Numer. Math. 2006, 56, 80-90. [CrossRef]

4. Parvizi, M.; Eslahchi, M.R.; Dehghan, M. Numerical solution of fractional advection-diffusion equation with a nonlinear source term. Numer. Algorithms 2015, 68, 601-629. [CrossRef]

5. Bu, W.; Liu, X.; Tang, Y.; Yang, J. Finite element multigrid method for multi-term time fractional advection diffusion equations. Int. J. Model. Simul. Sci. Comput. 2015, 6, 1540001. [CrossRef]

6. Donatelli, M.; Mazza, M.; Serra-Capizzano, S. Spectral analysis and structure preserving preconditioners for fractional diffusion equations. J. Comput. Phys. 2016, 307, 262-279. [CrossRef]

7. Donatelli, M.; Mazza, M.; Serra-Capizzano, S. Spectral analysis and multigrid methods for finite volume approximations of space-fractional diffusion equations. SIAM J. Sci. Comput. 2018, 40, A4007-A4039. [CrossRef]

8. Lin, X.L.; Ng, M.K.; Sun, H.W. A multigrid method for linear systems arising from time-dependent two-dimensional space-fractional diffusion equations. J. Comput. Phys. 2017, 336, 69-86. [CrossRef]

9. Shah, R.; Khan, H.; Kumam, P.; Arif, M. An Analytical Technique to Solve the System of Nonlinear Fractional Partial Differential Equations. Mathematics 2019, 7, 505. [CrossRef]

10. Khan, H.; Shah, R.; Baleanu, D.; Kumam, P.; Arif, M. Analytical Solution of Fractional-Order Hyperbolic Telegraph Equation, Using Natural Transform Decomposition Method. Electronics 2019, 8, 1015. [CrossRef]

11. Goswami, A.; Singh, J.; Kumar, D.; Gupta, S. An efficient analytical technique for fractional partial differential equations occurring in ion acoustic waves in plasma. J. Ocean Eng. Sci. 2019, 4, 85-99. [CrossRef]

12. Khan, Y.; Taghipour, R.; Falahian, M.; Nikkar, A. A new approach to modified regularized long wave equation. Neural Comput. Appl. 2013, 23, 1335-1341. [CrossRef]

13. Peregrine, D.H. Calculations of the development of an undular bore. J. Fluid Mech. 1966, 25, 321-330. [CrossRef]

14. Benjamin, T.B.; Bona, J.L.; Mahony, J.J. Model equations for long waves in nonlinear dispersive systems. Philos. Trans. R. Soc. Lond. Ser. A Math. Phys. Sci. 1972, 272, 47-78. [CrossRef]

15. Khalifa, A.K.; Raslan, K.R.; Alzubaidi, H.M. Numerical study using ADM for the modified regularized long wave equation. Appl. Math. Model. 2008, 32, 2962-2972. [CrossRef]

16. Achouri, T.; Omrani, K. Numerical solutions for the damped generalized regularized long-wave equation with a variable coefficient by Adomian decomposition method. Commun. Nonlinear Sci. Numer Simul. 2009, 14, 2025-2033. [CrossRef]

17. İdris, D. Least-squares quadratic B-spline finite element method for the regularised long wave equation. Comput. Methods Appl. Mech. Eng. 2000, 182, 205-215.

18. Bota, C.; Căruntu, B. Approximate analytical solutions of the regularized long wave equation using the optimal homotopy perturbation method. Sci. World J. 2014, 2014, 721865. [CrossRef]

19. Ganji, D.D.; Tari, H.; Jooybari, M.B. Variational iteration method and homotopy perturbation method for nonlinear evolution equations. Comput. Math. Appl. 2007, 54, 1018-1027. [CrossRef]

20. Inc, M.; Uğurlu, Y. Numerical simulation of the regularized long wave equation by He's homotopy perturbation method. Phys. Lett. A 2007, 369, 173-179. [CrossRef]

21. Noor, M.A.; Mohyud-Din, S.T. Modified variational iteration method for heat and wave-like equations. Acta Appl. Math. 2008, 104, 257-269. [CrossRef]

22. Noor, M.A.; Mohyud-Din, S.T. Variational homotopy perturbation method for solving higher dimensional initial boundary value problems. Math. Probl. Eng. 2008, 2008, 696734. [CrossRef]

23. Mohammad Mehdi Hosseini, S.; Tauseef Mohyud-Din, S.; Ghaneai, H. Variational iteration method for Hirota-Satsuma coupled KdV equation using auxiliary parameter. Int. J. Numer. Methods Heat Fluid Flow 2012, 22, 277-286. [CrossRef] 
24. Mohyud-Din, S.T.; Yildirim, A.; Hosseini, M.M. Variational iteration method for initial and boundary value problems using He's polynomials. Int. J. Differ. Equ. 2010, 2010, 426213. [CrossRef]

25. Liu, Y. Variational homotopy perturbation method for solving fractional initial boundary value problems. In Abstract and Applied Analysis; Hindawi: London, UK, 2012; Volume 2012.

26. Shah, R.; Khan, H.; Baleanu, D. Fractional Whitham-Broer-Kaup Equations within Modified Analytical Approaches. Axioms 2019, 4, 125. [CrossRef]

27. Shah, R.; Khan, H.; Farooq, U.; Baleanu, D.; Kumam, P.; Arif, M. A New Analytical Technique to Solve System of Fractional-Order Partial Differential Equations. IEEE Access 2019, 7, 150037-150050. [CrossRef]

28. Shah, R.; Khan, H.; Arif, M.; Kumam, P. Application of Laplace-Adomian Decomposition Method for the Analytical Solution of Third-Order Dispersive Fractional Partial Differential Equations. Entropy 2019, 21, 335. [CrossRef]

29. Mahmood, S.; Shah, R.; Arif, M. Laplace Adomian Decomposition Method for Multi Dimensional Time Fractional Model of Navier-Stokes Equation. Symmetry 2019, 11, 149. [CrossRef]

30. Khan, H.; Shah, R.; Baleanu, D.; Arif, M. An Efficient Analytical Technique, for The Solution of Fractional-Order Telegraph Equations. Mathematics 2019, 7, 426. [CrossRef]

31. Shah, K.; Khalil, H.; Khan, R.A. Analytical solutions of fractional order diffusion equations by natural transform method. Iran. J. Sci. Technol. Trans. A Sci. 2018, 42, 1479-1490. [CrossRef]

(C) 2020 by the authors. Licensee MDPI, Basel, Switzerland. This article is an open access article distributed under the terms and conditions of the Creative Commons Attribution (CC BY) license (http://creativecommons.org/licenses/by/4.0/). 